\title{
Electrophysiological parameters in anxiety and failure: Evaluation of doxepin and hydroxyzine
}

\author{
VLADIMIR PISHKIN and JAY T. SHURLEY \\ Behavioral Sciences Laboratories, Veterans Administration Medical Center, and \\ Department of Psychiatry and Behavioral Sciences, University of Oklahoma Health Sciences Center \\ Oklahoma City, Oklahoma 73104
}

\begin{abstract}
This double-blind investigation compared the effects of doxepin, hydroxyzine $\mathrm{HCl}$, and placebo on muscle action potentials (MAPs) and skin conductance (GSR) parameters as functions of experiencing either success or failure in concept-identification performance of 30 psychiatric anxiety patients. Results showed the following: (1) increase in MAPs with failure, (2) reduction of MAPs by both medications, (3) a greater number of spontaneous GSRs in the success condition compared with the failure condition, and (4) a significant negative relationship between MAPs and GSRs.
\end{abstract}

Cognitive deficit has been demonstrated by introducing antecedent failure (insoluble problem) to normal (Pishkin, 1965; Pishkin \& Shurley, 1968) as well as psychiatric (Pishkin, Fishkin, Shurley, Lawrence, \& Lovallo, 1978; Pishkin, Shurley, \& Wolfgang, 1967) populations. This type of deficit is especially evident in the highanxiety normal population with antecedent failure when compared to the low-anxiety group (Meites, Pishkin, \& Bourne, 1981). The experience of cognitive failure has been shown to produce a state of stress that is also reflected by psychophysiological parameters (Pishkin, Fishkin, \& Shurley, 1979). Moreover, experimentally manipulated motivation and drive-level factors interact with the success/failure dimension to further inhibit cognitive performance (Pishkin, Wolfgang, \& Bradshaw, 1963).

Both doxepin (Krakowski, 1968) and hydroxyzine HC1 (Pishkin et al., 1967) reduce anxiety with minimal lessening of cognitive abilities (Pishkin et al., 1978). There is evidence that hydroxyzine is also effective in relief of anxiety and tension states in adults as well as children (Feinberg, Pruzansky, Feinberg, \& Fisherman, 1958; Nathan \& Andelman, 1957; Sanchez-Ibanez, 1957). Hughes and Kopmann (1960) demonstrated that hydroxyzine was effective in reducing conditioned avoidance behavior without lessening discriminative capacity of rats. Doxepin is a tricyclic compound with chemical resemblance to amitriptyline, from which it differs by the presence of an oxygen atom in the central ring. Doxepin also differs from the other tricyclic amines

The authors wish to express their appreciation to James C. Spalding, M.D., for his valuable assistance in patient selection and administration of the drug regimen. The Laboratories Division, Pfizer Corporation, New York, supplied the drugs utilized. Send reprint requests to Vladimir Pishkin, Ph.D., Behavioral Sciences Laboratories (151A), Veterans Administration Medical Center, 921 N.E. 13th Street, Oklahoma City, Oklahoma 73104. in that it possesses both antianxiety and antidepressant properties (Goldberg \& Finnerty, 1972). Doxepin is effective in treating patients with a variety of disorders, including alcoholism, allergy management, severe emotional disturbances associated with physical illness, sleep disturbances, and treatment of anxiety neuroses (Ayd, 1969; Karacan, Blackburn, Thornby, Okawa, Salis, \& Williams, 1975). Accordingly, this study was designed to evaluate the comparative effects of doxepin and hydroxyzine upon cognitive stress (success/failure paradigm) that increases arousal levels as manifested by electrodermal (GSR) and electromyographic (EMG) activities of anxiety patients.

\section{METHOD}

\section{Subjects}

The subjects were 30 volunteer, newly admitted, first psychiatric admission patients at the Veterans Administration Medical Center, Oklahoma City, Oklahoma. All subjects had a primary diagnosis of anxiety disorder. The selection criteria were as follows: (1) Patients with CNS pathology or a history of either acute or chronic brain syndrome as specified in earlier work (Pishkin \& Burn, 1971) were excluded; (2) all patients had been free of psychotropic medication for at least 15 days before the beginning of the study; (3) each patient had a complete medical and psychiatric examination, a thorough physical examination, and a mental status and clinical examination by the admitting psychiatrist prior to diagnosis and admission to the project; (4) subjects ranged in age from 25 to 50 years (mean $=37.61$ years); and (5) all participants signed an informed consent form (VA-10-1086).

Twenty of the patients were given either doxepin or hydroxyzine, the remaining 10 (control) patients were given placebo (lactose) during the investigation. The medication was administered in identical capsules and contained either $20 \mathrm{mg}$ of doxepin or $50 \mathrm{mg}$ of hydroxyzine. The dosage administered was one capsule 3 times/day for 2 weeks. At the end of the 15-day period, the subjects performed on a concept-identification (CI) task. The stimuli were geometric patterns projected on a screen in front of the subject. The subject's task was to categorize the patterns in accordance with a relevant dimension by pressing one of the two response keys. The feedback was automatically pro- 
vided by amber lights above the keys. A more detailed description of the CI task and apparatus was provided in an earlier report (Pishkin \& Wolfgang, 1964). The CI problems were designed to elicit an expectation on the part of the subject of either success or failure. In the success condition, subjects received correct feedback on all trials; in the failure condition, they received 50\% misinformation (Pishkin, 1960), making it impossible to reach solutions. This misinformation condition has been shown to evoke an experience of failure and to reliably inhibit performance on subsequent cognitive tasks (Pishkin, 1965).

\section{Procedure}

The GSRs and EMGs were recorded concurrently with CI performance. The GSRs were recorded as DC resistance changes and converted to conductance measure. The active electrode was a $.625 \times 1.0 \mathrm{in}$. silver plate electrolytically coated with $\mathrm{AgCl}$ anodized in $1-\mathrm{M} \mathrm{NaCl}(3 \mathrm{~mA}$ for $5 \mathrm{~min})$. The EMGs were taken from the forehead utilizing frontalis placement specified by Davis (1952). The muscle action potentials (MAPs) were taken six times at the end of each 5-min interval, representing a 120 msec segment; the greatest amplitude within each segment was utilized in analysis of the data. The conductance change was computed in accordance with Edelberg's (1967) method: $\Delta C=\Delta R / R^{2}$.

The $\Delta R$ is resistance change, and $R^{2}$ is the basal resistance squared at the onset of the response. The spontaneous GSR measures consisted of the number of GSRs within six of the 5-min segments. Only the GSRs that were equal to or greater than .001 of basal resistance were counted as spontaneous GSRs in the analysis of the data. The subjects performed for $30 \mathrm{~min}$ on either success or failure CI task measures. A more detailed description of the psychophysiological procedures was reported earlier (Pishkin \& Shurley, 1968).

\section{Design}

This was a 2 by 3 factorial design with two CI problems (success or failure) and three drug conditions (doxepin, hydroxyzine $\mathrm{HCl}$, or placebo). Since the two-choice CI task was a relatively simple problem (three irrelevant dimensions), no difference in performance between the drug groups was expected in the success set condition (Pishkin et al., 1967), and, obviously, with $50 \%$ misinformation feedback, no learning could occur in the failure condition.

\section{RESULTS AND DISSCUSSION}

As anticipated, by design, there was no difference in CI performances between the drug groups in either the success or the failure condition $(\mathrm{Fs}<1.0)$. All subjects in the success condition solved the problem within the first $21 \mathrm{~min}$, and the failure subjects performed at the chance level, matching 50\% misinformation feedback throughout the 30-min period. Two independent analyses of variance were performed on the EMG and the GSR data.

Significantly greater degree of MAP was found in the failure conditions across the three drug groups as compared to the success condition $[F(1,24)=8.14$, $\mathrm{p}<.01]$. The main effect of drugs on MAP was also significant $[F(1,24)=6.07, p<.05]$, revealing the greatest amount of muscle tension in the placebo group and the least tension in the hydroxyzine condition. It is noteworthy that the placebo group produced the highest level of MAP $($ mean $=29.03)$, doxepin the middle level $($ mean $=20.41)$, and hydroxyzine the lowest $($ mean $=11.52)$. The EMG data are displayed in Figure 1.

The spontaneous GSR data revealed that only the main effect of success/failure was reliable $[F(1,24)=7.32$, $\mathrm{p}<.01]$. There was no main effect of drugs; neither was the interaction of Drug by Success/Failure significant (See Figure 2).

An overall significant negative correlation was found between spontaneous GSRs and MAPs $(r=-.61, p<.01)$, suggesting that the two measures tap different types of autonomic activation (i.e., more MAP is associated with failure than success, whereas increase in GSR activities reflects a state of experiencing success). This interpretation is consistent with earlier findings for which it was proposed that MAP concomitant with $\mathrm{CI}$ is an indicator of autonomic, internal disturbance associated with inability to process information, whereas spontaneous GSRs reflect successful intake of information (Pishkin \& Shurley, 1968). Apparently, this trend is significantly accentuated in anxiety patients, as demonstrated in this experiment.

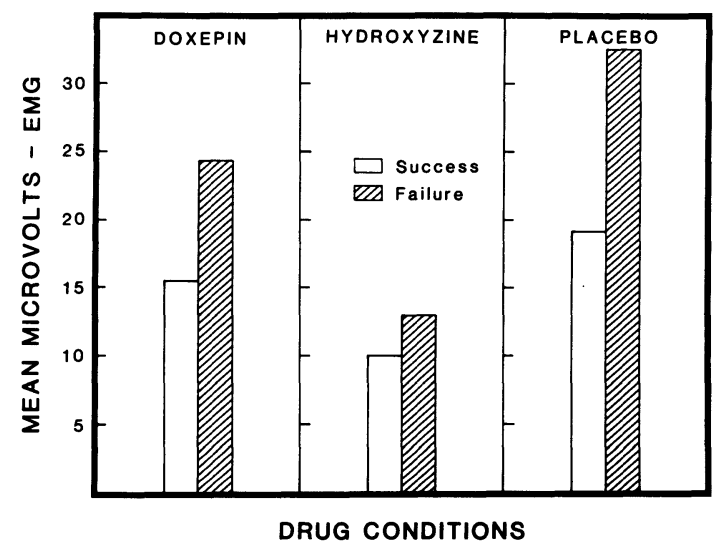

Figure 1. Muscle action potential activity as a function of success/failure manipulation and the three drug conditions.

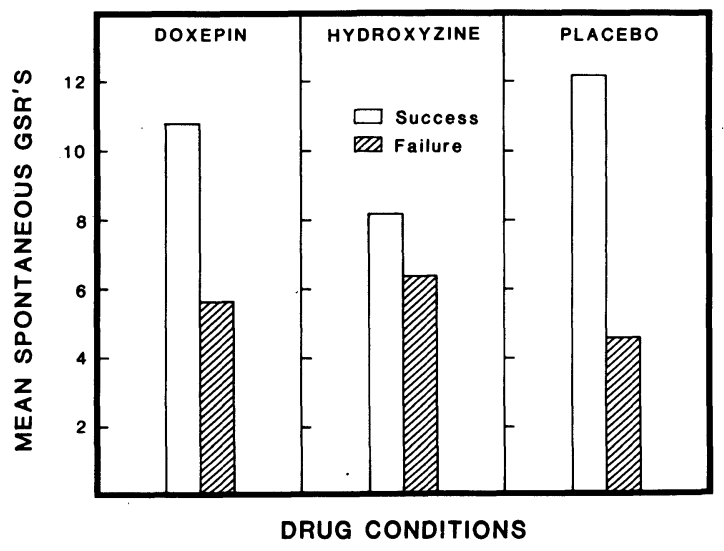

Figure 2. Spontaneous changes in skin conductance as functions of success/failure manipulation and the three drug conditions. 
It is noteworthy that hydroxyzine and, to a lesser degree, doxepin suppressed the muscle tension that was produced by the stress of experiencing failure when compared to the placebo. Moreover, it was demonstrated that CI failure is reliably related to an increase in muscle tension of clinically anxious subjects. If our speculation that spontaneous GSRs represent successful information intake is valid, then it may be concluded that the medications used in this study should have no detectable influence on cognitive performance. Most important, this study confirms an earlier finding with normal college subjects, in which high-anxiety subjects, as determined by the Taylor (1956) Manifest Anxiety Scale, performed better on the CI task following a success condition than they did following a failure condition (Meites et al., 1981). The success and failure conditions in the present experiment and the cited experiment were identical.

On the whole, a reliable relationship was shown between success/failure manipulation and the two electrophysiological parameters utilized. Furthermore, hydroxyzine reduced failure-induced muscle tension of the anxiety reaction patients. Future research is needed to explore how nonanxious subjects, under the cognitive stress of failure, would respond autonomically when medicated by the two frequently prescribed drugs that were utilized in the present study.

\section{REFERENCES}

Ayd, F. J. A clinical evaluation of doxepin (Sinequan). Diseases of the Nervous System, 1969, 30, 396-400.

DAvis, J. F. Manual of surface electromyography. Montreal: Laboratory for Psychological Studies, Allan Memorial Institute of Psychiatry, 1952.

EDELBERG, R. Electrical properties of the skin. In C. C. Brown (Ed.), Methods in psychophysiology. Baltimore, Md: Williams \& Wilkins, 1967.

Feinberg, A. R., Pruzansky, J. J. Feinberg, S. M., \& Fisherman, E. W. Hydroxyzine (Atarax) in chronic urticaria and in allergic manifestations. Journal of Allergy, 1958, 29, 358.

Goldberg, H. I., \& Finnerty, R. J. The use of doxepin in the treatment of symptoms of anxiety neurosis and accompanying depression: A collaborative controlled study. American Journal of Psychiatry, 1972, 129, 47-77.

Hughes, F. W., \& KopmanN, E. Influence of pentobarbital, hydroxyzine, chlorproinazine, reserpine, and meprobamate on choice-discrimination behavior in the rat. Archives Internationales de Pharmacodynamine, 1960, 126, 158-170.

Karacan, I., Blackburn, A. B., Thornby, J. I., Okawa, M., Salis, P. J., \& Williams, R. L. The effect of doxepin $\mathrm{HCl}$ (Sinequan) on sleep patterns and clinical symptomatology of neurotic depressed patients with sleep disturbance. In J. Mendels (Ed.), Sinequan (doxepin $\mathrm{HCl}$ ): $\mathrm{A}$ monograph of recent clinical studies. Amsterdam: Excerpta Medica, 1975.

KrAKowsKi, A. J. Activity study of doxepin-A new antidepressant. Psychosomatics, 1968, 9, 89-95.

Meites, K., Pishkin, V., \& Bourne, L. E., Jr. Anxiety and failure in concept identification. Bulletin of the Psychonomic Society, 1981, 18, 293-295.

Nathan, L. A., \& Andelman, M. B. The use of tranquilizer in the management of behavior problems in a private pediatric practice. Illinois Medical Journal, 1957, 112, 171-172.

Pishkin, V. Effects of probability of misinformation and number of irrelevant dimensions upon concept identification. Journal of Experimental Psychology, 1960, 59, 371-378.

Pishkin, V. Dimension availability with antecedent success or failure in concept identification. Psychonomic Science, 1965, 2, 69-70.

Pishkin, V., \& Burn, J. M. Concept identification in the braindamaged: Intertrial interval and information complexity. Journal of Abnormal Psychology, 1971, 77, 205-210.

Pishkin, V., Fishkin, S. M., \& Shurley, J. T. Motivation in cognitive performance: Double-blind comparison of chlordiazepoxide with doxepin. Current Therapeutic Research, 1979, 25, 165-171.

Pishkin, V., Fishkin, S. M., Shurley, J. T., Lawrence, B. E., \& Lovallo, W. R. Cognitive and psychophysiologic response to doxepin and chlordiazepoxide. Comprehensive Psychiatry, 1978, 2, 171-178.

Pishinin, V., \& Shurley, J. T. Electrodermal and electromyographic parameters in concept identification. Psychophysiology, $1968,5,112-118$.

Pishkin, V., Shurley, J. T., \& Wolfang, A. Stress: Psychophysiological and cognitive indices in an acute double-blind study with hydroxyzine in psychiatric patients. Archives of General Psychiatry, 1967, 16, 471-478.

Pishoin, V., \& Wolfana, A. Electromyographic gradients in concept identification with numbers of irrelevant dimensions. Journal of Clinical Psychology, 1964, 20, 61-67.

Pishkin, V., Wolfgang, A., \& Bradshaw, F. J., Jr. Hydroxyzine in concept identification under induced stress with chronic schizophrenics. Journal of Nervous and Mental Disease, 1963, 137, 322-328.

Sanchez-Ibanez, J. M. Hydroxyzine hydrochloride in labor. Revista IBYS-Instituto de Biologia y Sueroterapia, 1957, 4, 143-149.

TAylor, J. Drive theory and manifest anxiety. Psychological Bulletin, 1956, 53, 243-244.

(Received for publication November 29, 1982.) 